\title{
Glücklich mit künstlicher Intelligenz und Big Data?
}

\author{
Jean Martin
}

Dr. med., Mitglied der Redaktion

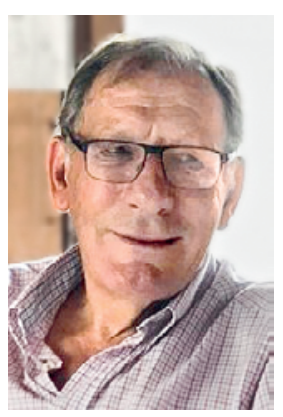

Die Ethikgruppe Cèdres Réflexion organisiert in diesem Jahr eine Vortragsreihe zum Thema Glück. Ein Vortrag trug kürzlich den Titel «Glück nach Google?». Referenten waren Solange Ghernaouti von der Universität Lausanne, sie ist vor allem bekannt für ihre Arbeit im Bereich Cybersicherheit, der Informatiker Jean-Gabriel Ganascia, Vorsitzender der Ethikkommission des CNRS in Paris, und François Fleuret, er erforscht die künstliche Intelligenz am Institut Idiap-EPFL (Martigny).

Diese Debatte zum Thema digitale Technologien war interessant. Gewiss, man kann die "Internetgiganten» (die GAFA) dafür kritisieren, dass sie kostenlos riesige Mengen unserer Daten abgreifen und daraus Informationen ableiten. Würden sie dies jedoch nicht tun, wären diese Daten überall verstreut, ohne Nutzen zu bringen! Fleuret: «Big Data allein kann die Welt nicht verstehen, aber die daraus ableitbaren Analysen ermöglichen häufig zuverlässige und sinnvolle Prognosen und Einschätzungen.» Ghernaouti zum Thema Datenbanken mit unseren biologischen Daten: «Hier werden unserem Körper Informationserze entnommen.»

Ein grosses Plus des Internets: (Fast) alles ist kostenlos. Jeder Nutzer (auch der arme und benachteiligte) hat weltweiten Zugang zum gesamten Wissen der Menschheit. Big Data ermöglicht Fortschritte für seltene Krankheiten. Wo sind die Fallstricke? Wir erleben eine unglaubliche Machtkonzentration bei den GAFA, die Auswirkungen auf die Privatsphäre bereiten grosse Sorge, Internetsucht wird immer mehr zum Thema auch für die WHO. Die Geissel der Fake News wird durch die sozialen Netze noch mehr aufgebläht. Ganascia äussert die Befürchtung, dass wir - wenn wir die Karotte der GAFA annehmen - letztlich die Esel sind ...

Weitere Nebenwirkungen: Wir interagieren nicht mehr direkt miteinander. Wir müssen (wieder) lernen, einander in die Augen zu schauen (und nicht nur auf das Smartphone). Ein grosser Grund zur Sorge ist, dass das Prinzip der Gegenseitigkeit in unserer Gesellschaft verloren geht, beispielsweise bei der Krankenversicherung. Wie lange noch bleibt uns das Solidaritätsprinzip (wie z.B. bei der KVG) erhalten, wenn wir die Risikofaktoren jedes Einzelnen und dessen wahrscheinliche medizinische Entwicklung im Handumdrehen ermitteln? Zum
Thema allfällige Korrektur- bzw. Umkehrmassnahmen merkt einer der Redner an, dass Asbest, ein beliebter Isolierstoff Mitte des 20. Jahrhunderts, der sich als sehr gesundheitsschädlich erwiesen hatte, zwar erfolgreich aus dem Verkehr gezogen werden konnte, es aber weitaus schwieriger sei, die Gesellschaft zu «entcomputerisieren".

Kategorisch versichern wir uns, dass die künstliche Intelligenz niemals an den menschlichen Geist heranreichen wird. Fleuret hat da seine Zweifel. Er sieht keine "computational wall», die unüberbrückbar wäre. Warum sollte es da einen Schwelleneffekt geben? Wir gehen kontinuierlich, Schritt für Schritt, voran. Analog lässt es sich in Bezug auf den Transhumanismus argumentieren. Viele wehren sich kategorisch gegen den Gedanken, vergessen dabei aber, dass in der Realität die Grenze zwischen dem natürlichen und dem "optimierten» Menschen sehr durchlässig ist (viele von uns tragen Brillen oder Prothesen; es gibt eine kontinuierliche Weiterentwicklung, die sich immer technologischer und intrusiver gestaltet).

Und wie sollen wir uns gegen die makroökonomischen und makropolitischen Derivate wappnen? Die Netz-Multis stellen eine gewaltige Macht dar. Die Nationalstaaten haben sie im Laufe der letzten Jahrzehnte gewähren lassen und sehen sich heute vor der Schwierigkeit, sie in ihre Schranken verweisen und ihnen Verhaltenskodizes aufoktroyieren zu wollen (ausser in China?).

Schlussfolgerungen? Natürlich bergen technologische Entwicklungen ein grosses Potential. Keiner der Redner hat sich jedoch in Bezug auf unsere hypervernetzte Zukunft an präzise Vorhersagen gewagt. Zu komplex ... zu abhängig von multiplen, mehr oder weniger greifbaren Parametern. Sie glauben nicht, dass Technologien die heutige Welt "verzaubern» könnten, obwohl die Welt sie eigentlich braucht. Ghernaouti zitiert einen Kollegen: «Was ich im Leben nicht gefunden habe, habe ich auch im Netz nicht gefunden.»

Die Debatte hat natürlich nicht wirklich auf die Frage geantwortet, ob Google uns glücklich machen könnte. Dazu müssten wir uns zunächst darüber einig werden, was wir unter Glück verstehen - eine weitere schwierige Frage! 